\title{
ON THE DUNFORD-PETTIS PROPERTY IN SPACES OF VECTOR-VALUED BOUNDED FUNCTIONS
}

\author{
Manuel D. Contreras and Santiago díaz
}

We show that $L^{\infty}(\mu, X)$ has the Dunford-Pettis property for some classical Banach spaces including $L^{1}(\mu), C(K)$, the disc algebra $A$ and $H^{\infty}$.

A Banach space $X$ is said to have the Dunford-Pettis property if every weakly compact operator from $X$ into an arbitrary Banach space is completely continuous, or equivalently, if given sequences $\left(x_{n}\right)$ in $X$ and $\left(x_{n}^{*}\right)$ in $X^{*}$, both weakly convergent to zero, then $\left\langle x_{n}^{*}, x_{n}\right\rangle$ tends to zero. A detailed exposition about this property can be found in [6]. In this reference, the following problem is posed [6, p.55]: assume that $L^{\infty}(\mu, X)$ denotes the Banach space of (equivalence classes of) essentially bounded, measurable and $X$-valued functions defined over a finite measure space $(\Omega, \Sigma, \mu)$. When does $L^{\infty}(\mu, X)$ have the Dunford-Pettis property? In general, this property does not lift from $X$ to $L^{\infty}(\mu, X)[6, \mathrm{p} .56]$. On the other hand, the only non-trivial positive result, as far as we know, is that $L^{\infty}\left(\mu, L^{1}(\nu)\right)$ has the property when $\mu$ is purely atomic [3, Theorem 1]. The aim of this note is to provide some new positive examples. Namely, we show that $L^{\infty}(\mu, X)$ has the Dunford-Pettis property for every arbitrary finite measure $\mu$, whenever $X$ is either any $\mathcal{L}^{1}$-space or any $\mathcal{L}^{\infty}$-space or the disc algebra or the space $H^{\infty}$ of bounded analytic functions on the disc.

To avoid trivial situations, we always assume that there exists a pairwise disjoint sequence $\left(C_{m}\right)$ in $\Sigma$ such that $\mu\left(C_{m}\right)>0$. The notation is standard except, perhaps, the following one: if $\left(A_{m}\right)$ is a sequence of pairwise disjoint $\Sigma$-measurable subsets of non-zero measure, we write

$$
\left[A_{m}\right]:=\left\{\sum_{m=1}^{\infty} \chi_{A_{m}}(\cdot) x_{m}, \quad\left(x_{m}\right) \in \ell^{\infty}(X)\right\} .
$$

It is well-known that $\left[A_{m}\right]$ is a complemented subspace of $L^{\infty}(\mu, X)$ isometrically isomorphic to $\ell^{\infty}(X)$. In particular, if $L^{\infty}(\mu, X)$ has the Dunford-Pettis property,

Received 28th March, 1995

This research has been partially supported by La Consejería de Educación y Ciencia de la Junta de Andalucía.

Copyright Clearance Centre, Inc. Serial-fee code: 0004-9729/96 \$A2.00+0.00. 
then $\ell^{\infty}(X)$ has it and our main aim (Theorem 1 below) is to prove that the converse holds. Similar ideas have been used in [4].

We refer the reader for the terminology used here to the monographs of Diestel and Uhl [7], Lindenstrauss and Tzafriri [8] and Wojtaszczyk [10].

Theorem 1. $L^{\infty}(\mu, X)$ has the Dunford-Pettis property if (and only if) $\ell^{\infty}(X)$ has it.

Proof: Suppose that $\left(f_{n}\right)$ and $\left(f_{n}^{*}\right)$ are sequences in $L^{\infty}(\mu, X)$ and $L^{\infty}(\mu, X)^{*}$ respectively, both weakly convergent to zero. We have to prove that $\left\langle f_{n}^{*}, f_{n}\right\rangle$ tends to zero.

The proof of Pettis's Measurability Theorem, as can be seen in [7, Chapter 2, Theorem 2], shows that, for each $n \in \mathbb{N}$, there are a bounded sequence $\left(x_{m}(n)\right)_{m}$ in $X$ and a sequence $\left(A_{m}(n)\right)_{m}$ of pairwise disjoint $\Sigma$-measurable subsets with positive measure covering $\Omega$, such that if

$$
g_{n}(\cdot):=\sum_{m=1}^{\infty} x_{m}(n) \chi_{A_{m}(n)}(\cdot) \in L^{\infty}(\mu, X)
$$

we have $\left\|f_{n}-g_{n}\right\| \leqslant 1 / n$. Hence, it is enough to prove that $\left\langle f_{n}^{*}, g_{n}\right\rangle$ tends to zero.

Denote by $X_{n}$ the finite-dimensional subspace of $L^{\infty}(\mu, X)$ spanned by $g_{1}, \ldots, g_{n}$. Furthermore, for every $n \in \mathbb{N}$, consider the following family of pairwise disjoint $\Sigma$ measurable subsets of $\Omega$ :

$$
\mathcal{A}^{n}:=\left\{A_{m_{1}}(1) \cap A_{m_{2}}(2) \cap \ldots \cap A_{m_{n}}(n): \quad m_{1}, m_{2}, \ldots, m_{n} \in \mathbb{N}\right\}
$$

Arrange $\mathcal{A}^{n}$ in a sequence $\left(B_{m}^{n}\right)_{m}$. It is easy to see that $X_{n}$ is included in $Y_{n}=\left[B_{m}^{n}\right]$. Since $\left(Y_{n}\right)$ is increasing, the closure $Y$ of $\bigcup_{n} Y_{n}$ is a closed subspace of $L^{\infty}(\mu, X)$ and $g_{n}$ is a weakly null sequence in $Y$. On the other hand, the restrictions $f_{n \mid Y}^{*}$ also form a weakly null sequence in $Y^{*}$. To finish the proof, we only have to show that $Y$ has the Dunford-Petis property. According to a result due to Bourgain [3, Proposition 2], it is enough to prove that $\left(\oplus_{n} Y_{n}\right)_{\infty}$ has the Dunford-Pettis property. But, this follows by combining the hypothesis that $\ell^{\infty}(X)$ has the Dunford-Pettis property with the following topological isomorphisms:

$$
\left(\oplus_{n} Y_{n}\right)_{\infty} \cong\left(\oplus_{n} \ell^{\infty}(X)\right)_{\infty} \cong \ell^{\infty}(X)
$$

We need later the following variant of Theorem 1 which is of independent interest.

TheOREM 2. Assume that given a sequence of finite-dimensional subspaces $\left(X_{n}\right)$ in $\ell^{\infty}(X)$, we can find, for each $n$, another sequence of subspaces $\left(X_{n, m}\right)_{m}$ in $X$, such 
that $X_{n, m} \subset X_{n+1, m}, X_{n} \subset Y_{n}=\left(\oplus_{m} X_{n, m}\right)_{\infty}$ and $\left(\oplus_{n} Y_{n}\right)_{\infty}$ has the Dunford-Pettis property. Then, $L^{\infty}(\mu, X)$ has it.

Proof: Arguing, as in the proof above, one shows that $\ell^{\infty}(X)$ has the DunfordPettis property. Then, applying Theorem 1 , we obtain that $L^{\infty}(\mu, X)$ has this property.

REMARK. Theorem 1 shows that the measure $\mu$ does not play a significant role. This is in contrast to other vector-valued situations. Indeed, the behaviour of purely atomic and atomless measures with respect to the Dunford-Pettis property is quite different in $L^{1}(\mu, X)$ (see $[2$, Corollary $2.4(\mathrm{c})]$ and [9, Théorème 3]). Something similar happens with perfect and dispersed compacts in $C(K, X)$ (see [1, Theorem 2] and [9, Théorème 3]). It is worth mentioning that, in general, $\ell^{\infty}(X)$ and $L^{\infty}(\mu, X)$ are not isomorphic [5, Corollary 1].

We recall that a Banach space $X$ is said to be an $\mathcal{L}^{p}$-space $(1 \leqslant p \leqslant \infty)$, in the sense of Lindenstrauss-Petczyniski, if there is $\lambda \geqslant 1$ such that, for every finitedimensional subspace $Y$ of $X$, there is another finite-dimensional subspace $Z$ of $X$ such that $Y$ is contained in $Z$ and $d\left(Z, \ell_{k}^{p}\right) \leqslant \lambda$, for some $k \in \mathbb{N}$.

Corollary 1 . Denote by $X$ either any $\mathcal{L}^{1}$-space or any $\mathcal{L}^{\infty}$-space. Then, $L^{\infty}(\mu, X)$ has the Dunford-Pettis property.

Proof: Bearing in mind the definition of $\mathcal{L}^{p}$-space and Theorem 2, it is not difficult to see that we only need to show that $\left(\oplus_{n} \ell_{r_{n}}^{p}\right)_{\infty}\left(r_{n} \in \mathbb{N} ; p=1, \infty\right)$ has the Dunford-Pettis property. The case $p=1$ follows from Bourgain's result [3, Theorem 1] and, for the case $p=\infty$, we note that $\left(\oplus_{n} \ell_{r_{n}}^{\infty}\right)_{\infty} \cong \ell^{\infty}$.

Corollary 2. Denote by $X$ either the disc algebra $A$, or the space of bounded analytic functions on the disc $H^{\infty}$. Then, $L^{\infty}(\mu, X)$ has the Dunford-Pettis property.

Proof: This follows directly from Theorem 1 and [4, proof of Theorem 2].

\section{REFERENCES}

[1] F. Bombal, 'On weakly compact operators on spaces of vector valued continuous functions', Proc. Amer. Math. Soc. 97 (1986), 93-96.

[2] F. Bombal, 'Distinguished subsets in vector sequence spaces', in Progress in Functional Analysis, (K.D. Bierstedt, J. Bonet, J. Horváth and M. Maestre, Editors) (North-Holland /Elsevier Science Publishers B.V., Amsterdam, New York, Oxford, 1992), pp. 293-306.

[3] J. Bourgain, 'On the Dunford-Pettis property', Proc. Amer. Math. Soc. 81 (1981), 265-272.

[4] M.D. Contreras and S.Diaz, ' $C(K, A)$ and $C\left(K, H^{\infty}\right)$ have the Dunford-Pettis property', Proc. Amer. Math. Soc. (to appear).

[5] S. Díaz, 'Complemented copies of $c_{0}$ in $L^{\infty}(\mu, X)$ ', Proc. Amer. Math. Soc. 120 (1994), 1167-1172. 
[6] J. Diestel, 'A survey of results related to the Dunford-Pettis property', in Conternporary Math., 2, Proc. of the Conf. on Integration, Topology and Geometry in Linear Spaces (Amer. Math. Soc., Providence, RI, 1980), pp. 15-60.

[7] J. Diestel and J.J. Uhl, Jr., Vector measures, Math. Surveys, 15 (Amer. Math. Soc., Providence, RI, 1977).

[8] J. Lindenstrauss and L. Tzafriri, Classical Banach spaces, Lecture Notes in Mathematics, 338 (Springer-Verlag, Berlin, Heidelberg, New York, 1973).

[9] M. Talagrand, 'La propriété de Dunford-Pettis dans $C(K, E)$ et $L_{1}(E)$ ', Israel J. Math. 44 (1983), 317-321.

[10] P. Wojtaszczyk, Banach Spaces for Analysts, Cambridge Studies in Advanced Mathematics, 25 (Cambridge University Press, Cambridge, 1991).

Departamento de Matemática Aplicada II

Universidad de Sevilla

E.S. Ingenieros Industriales

41012 Sevilla

Spain

e-mail: contreras@cica.es

madrigal@cica.es 